\title{
Non-Linear Poisson-Boltzmann Theory of a Wigner-Seitz Model for Swollen Clays
}

\author{
R. J. F. Leote de Carvalho ${ }^{1}$, E. Trizac ${ }^{2}$ and J.-P. Hansen ${ }^{3}$ \\ ${ }^{1}$ Department of Physics and Astronomy, University College London, \\ Gower Street, London WC1E 6BT, UK \\ and Laboratoire de Physique, Ecole Normale Supérieure de Lyon, (URA CNRS 1325) \\ 46, Allée d'Italie, 69364 Lyon Cedex 0\%, France \\ ${ }^{2}$ Laboratoire de Physique Théorique, UMR 8627 du CNRS, \\ Université Paris XI, Bâtiment 210, 91405 Orsay Cedex, France \\ ${ }^{3}$ Department of Chemistry, University of Cambridge \\ Lensfield Road, Cambridge CB2 1EW, UK
}

PACS. 82.70.Gg - Gels and sols

PACS. 02.60.Nm - Integral and integrodifferential equations

PACS. 68.10.-m - Fluid surfaces and fluid-fluid interfaces

\begin{abstract}
Swollen stacks of finite-size disc-like Laponite clay platelets are investigated within a Wigner-Seitz cell model. Each cell is a cylinder containing a coaxial platelet at its centre, together with an overall charge-neutral distribution of microscopic co and counterions, within a primitive model description. The non-linear Poisson-Boltzmann (PB) equation for the electrostatic potential profile is solved numerically within a highly efficient Green's function formulation. Previous predictions of linearised Poisson-Boltzmann (LPB) theory are confirmed at a qualitative level, but large quantitative differences between PB and LPB theories are found at physically relevant values of the charge carried by the platelets. A hybrid theory treating edge effect at the linearised level yields good potential profiles. The force between two coaxial platelets, calculated within PB theory, is an order of magnitude smaller than predicted by LPB theory
\end{abstract}

\section{INTRODUCTION}

Electrostatic interactions between suspended mesoscopic particles or polyions play a key role in determining the stability, mesostructure and phase behaviour of colloidal dispersions and polyelectrolytes. Polyions in aqueous dispersions may be rigid or flexible, and range in shape from spherical to rod-like or lamellar. Widely studied examples of polyions include polystyrene balls, elongated TMV (Tobacco Mosaic Virus) particles, stiff polyelectrolyte chains like DNA, flexible membranes and silicate clay platelets. The highly charged polyions strongly attract or repel microscopic counterions and coions (microions), leading to the formation of electric double layers characterised by highly inhomogeneous charge distributions around the polyions. Theoretical investigations of the structure of such double layers, and of their mutual effective interactions, have been mostly restricted to the simplest polyion topologies, including uniformly charged infinite planes or spheres. Starting with the pioneering work of Gouy [1] and Chapman [2], on infinite planar double-layers, theoretical calculations are almost invariably based on the mean-field Poisson-Boltzmann (PB) approximation, which neglects excluded volume and Coulomb correlations between the microions, although some recent attempts have been made to include such correlations within a density functional (DFT) formulation, which may also account for discrete solvent effects [3]. The non-linear PB equation for the local electrostatic potential may be solved analytically for a single planar double-layer, and two interacting double-layers in the salt-free case (i.e. in the absence of coions) [1,2., 4 ; in the presence of salt, the one-dimensional problem of interacting charged planes is easily solved numerically 15 . However, PB theory becomes increasingly difficult to handle for more complicated geometries, and must generally be linearised to become tractable. A well-known application of linearised PoissonBoltzmann (LPB) theory is the calculation of the effective interaction between colloidal spheres carrying a uniform surface charge, leading to the screened Coulomb potential of Derjaguin, Landau, Verwey and Overbeek [6,7] (DLVO). LPB theory is, however, strictly speaking valid only provided the local potential energy felt by the microions is everywhere small compared to the thermal energy $k_{B} T$. This condition is rarely met in the immediate vicinity of the 
highly-charged polyions, where the Coulomb energy becomes large compared to $k_{B} T$, so that non-linearities become crucial and full PB theory should be used to determine the concentration profiles of the microions. The deficiencies of LPB theory are frequently patched up by introducing the rather vague concept of counterion condensation, leading to a Helmholtz-Stern layer of counterions highly bound to the polyions [8], and henceforth to an effective valence $Z_{\text {eff }}$ of the latter, significantly reduced in magnitude compared to the nominal valence $Z$. It should be stressed that counterion condensation is a well-defined concept only within the cylindrical geometry of an infinite, uniformly charged thin rod [9,10]. In all other geometries, effective valences $Z_{\text {eff }}$ can only be defined within some phenomenological convention 11. 12]. In the case of spherical charge-stabilised colloids some recent direct measurements of the effective pair potential between polyions in the bulk of a suspension point to the validity of the functional form and range of the DLVO potential, provided $Z_{\text {eff }}$ is considered to be an adjustable parameter, varying within a physically reasonable range [13].

This paper deals with electric double-layers in lamellar stacks of uniformly charged finite platelets, considered as a model for swollen clays. Due to the finite size of the platelets, edge effects come into play so that the problem ceases to be one-dimensional, as would be the case for stacks of infinite planes. For simplicity, the clay platelets are assumed to be disc-shaped and coaxial, while they are equally spaced within an infinite stack. Fairly monodisperse disc-shaped platelets are synthetised as Laponite [14, a model clay which has received much recent experimental and theoretical attention. In particular a Wigner-Seitz (WS) model for such stacks has been thoroughly investigated within LPB theory [15,16]. Under most physical circumstances, the conditions of validity of LPB theory are far from being met in swollen clays, and consequently the much more difficult problem of non-linear PB theory for stacks of coaxial charged discs is being addressed in this paper.

The key innovation of the present work is that the solution of the non-linear partial differential equation for given boundary conditions is not sought within a standard finite difference scheme [17], but rather by expressing the solution in terms of the appropriate Green's function. The latter is obtained analytically for the present cylindrical geometry, in the form of a Bessel-Dini series, and the resulting non-linear integral equation is solved numerically by a very stable iterative procedure.

The remainder of the paper is organised as follows. The Wigner-Seitz model, appropriate for stacks of coaxial charged discs, is defined in section II. The basic assumptions and equations of PB theory are laid out in section III, and the Green's function methodology for solving the non-linear PB equation with cylindrical WS boundary conditions is presented in section IV. A hybrid PB/Debye-Hückel theory, whereby edge effects are treated to linear order in the deviation of the potential from its counterpart for infinite planar geometry, is formulated in section V. Numerical results for potential and concentration profiles, and for the resulting osmotic properties, are presented in section VI. The force acting between two finite, coaxial platelets is calculated in section VII, while conclusions are drawn in section VIII. A preliminary account of parts of this work was published elsewhere [18].

\section{WIGNER-SEITZ MODEL FOR SWOLLEN CLAYS}

Consider a stack of coaxial, infinitely thin, disc-shaped clay platelets, with an average spacing $H=2 h$. Each platelet has a radius $r_{0}$, and carries a uniform charge density $\sigma=-Z e / \pi r_{0}^{2}$, where $Z e$ is the total charge on a platelet. For Laponite, $r_{0} \approx 150 \AA$ and $Z \approx 1000$ 14]. Stacks are assumed to fill space in a columnar arrangement, with the normals to the platelets all pointing in the same direction. Each platelet is then placed, on average, at the centre of a Wigner-Seitz cell, of volume $v=V / N$, where $V$ is the total volume of the sample and $N$ the total number of platelets. In a hexagonal columnar array, the topology of the WS cell would be a prism of height $H$ and hexagonal basis parallel to the platelet. The WS model thus describes a regular three-dimensional array of platelets with perfectly aligned axes, and is thus appropriate to describe moderately swollen stacked clays, but would not be appropriate for dilute dispersions of clay particles. In view of the circular shape of the platelet it is reasonable to replace the WS prism by a cylinder of identical volume and height. In fact it was shown explicitly in Ref. 115] that, at least within LPB theory, the results are very insensitive to the precise shape of the WS cell. Compared to previous studies of stacked clay platelets, assumed to be infinite planes [5], the present study takes into account edge (or finite platelet size) effects.

The WS model for a swollen clay which will be examined in this paper reduces hence to a single charged circular platelet placed at the centre of a coaxial cylinder, together with $N_{+}$and $N_{-}$monovalent counterions and coions. Overall charge neutrality requires that

$$
N_{+}=N_{-}+Z .
$$

The molecular nature of the solvent (water) inside the cell will be ignored, i.e. the solvent is treated as a continuum of dielectric constant $\epsilon$ (primitive model of electrolytes). The height $H$ and radius $R>r_{0}$ of the cylinder must be 
such that $\pi R^{2} H=v$, where the volume $v$ is determined by the macroscopic concentration of clay platelets. The latter does not determine the aspect ratio $H / R$, which will be determined by the condition that it minimizes the total free energy of the microion distribution within the cell. The task is now to determine the total electrostatic potential $\varphi(\mathbf{r})$ throughout the cell, and to derive from it the concentration profiles $\rho^{+}(\mathbf{r})$ and $\rho^{-}(\mathbf{r})$ of counterions and coions, and the resulting osmotic properties, including the free energy, for a given volume $v$ and aspect ratio $H / R$. In keeping with the physical meaning of the WS cell as representing the cage formed by the neighboring platelets, the component of the electric field $\mathbf{E}$ normal to the surface $\Sigma$ of the cylinder will be assumed to vanish at each point of that surface, i.e. the following Neumann boundary condition will be imposed throughout

$$
[\mathbf{n}(\mathbf{r}) \cdot \nabla]_{\mathbf{r} \in \Sigma} \varphi(\mathbf{r})=0,
$$

where $\mathbf{n}(\mathbf{r})$ is the normal to the surface $\Sigma$ at $\mathbf{r}$. The task thus defined will be carried out within PB theory in the following sections.

\section{POISSON-BOLTZMANN THEORY.}

The electrostatic potential throughout the WS cell satisfies Poisson's equation

$$
\nabla^{2} \varphi(\mathbf{r})=-\frac{4 \pi}{\epsilon} \rho_{c}(\mathbf{r})
$$

where $\rho_{c}(\mathbf{r})$ is the total charge density, which includes the contribution of the platelet, $q_{P}(\mathbf{r})$, and that of the (monovalent) microions

$$
\rho_{c}(\mathbf{r})=q_{P}(\mathbf{r})+e\left[\rho^{+}(\mathbf{r})-\rho^{-}(\mathbf{r})\right] .
$$

In the mean-field approximation, the positions of microions are uncorrelated, so that their local concentrations are simply related to the local potential by the following Boltzmann distributions

$$
\rho^{ \pm}(\mathbf{r})=\rho_{0}^{ \pm} \mathrm{e}^{\mp \beta e \varphi(\mathbf{r})},
$$

where $\beta \equiv 1 / k_{B} T$. It is worth remembering that Eq. (5) is just a statement of the Euler-Lagrange equation resulting from the minimisation of the following free energy functional

$$
\begin{aligned}
\mathcal{F}\left[\rho^{+}(\mathbf{r}), \rho^{-}(\mathbf{r})\right] & =\mathcal{F}_{\text {ideal }}+\mathcal{F}_{\text {Coulomb }} \\
\mathcal{F}_{\text {ideal }} & =\sum_{\alpha=+,-} k_{B} T \int_{v} \rho^{\alpha}(\mathbf{r})\left[\ln \left(\Lambda_{\alpha}^{3} \rho^{\alpha}(\mathbf{r})\right)-1\right] d \mathbf{r} \\
\mathcal{F}_{\text {Coulomb }} & =\frac{1}{2} \int_{v} \rho_{c}(\mathbf{r}) \varphi(\mathbf{r}) d \mathbf{r},
\end{aligned}
$$

keeping in mind that $\rho_{c}(\mathbf{r})$ and $\varphi(\mathbf{r})$ are linearly related by Poisson's equation.

The prefactors $\rho_{0}^{ \pm}$in Eq. (5) have no physical significance when considered separately. However, their product $\rho_{0}^{+} \rho_{0}^{-}$is uniquely determined by the imposed physical conditions. In a canonical description, $N_{+}$and $N_{-}$are fixed by the macroscopic concentrations $n_{+} \equiv N_{+} / v$ and $n_{-} \equiv N_{-} / v$ of counterions and coions; the latter coincides with the concentration $n_{S}$ of added salt, i.e. $n_{-}=n_{S}$. The electroneutrality condition (1) then fixes $n_{+}$, and the prefactors $\rho_{0}^{ \pm}$are determined by the normalisation constraints

$$
n_{ \pm}=\frac{1}{v} \int_{v} \rho^{ \pm}(\mathbf{r}) d \mathbf{r} .
$$

Alternatively, if the suspension is in osmotic equilibrium with an ionic solution of concentration $n_{S}^{\prime}$ acting as a reservoir, a semi-grand-canonical description is in order. In this case the chemical potential of co and counterions must be identical in the suspension and in the reservoir, leading to the condition 胢

$$
\rho_{0}^{+} \rho_{0}^{-}=\left(n_{S}^{\prime}\right)^{2} .
$$

A key quantity describing the solution is then the Donnan ratio $n_{s} / n_{S}^{\prime}$ of salt concentrations in the cell and in the reservoir. Substitution of Eq. (5) into Eqs. (4) and (3) leads to the closed non-linear partial differential equation for the potential $\varphi(\mathbf{r})$ 


$$
\nabla^{2} \varphi(\mathbf{r})=-\frac{4 \pi}{\epsilon}\left(q_{P}(\mathbf{r})+e\left[\rho_{0}^{+} \mathrm{e}^{-\beta e \varphi(\mathbf{r})}-\rho_{0}^{-} \mathrm{e}^{\beta e \varphi(\mathbf{r})}\right]\right) .
$$

This PB equation must be solved subject to the Neumann boundary condition (2) on the surface of the WS cylinder. The linearised version of Eq. (9), where the Boltzmann factors are expanded to first order in $\varphi$ (LPB theory) was solved analytically in Refs. [15, 16], under the same boundary conditions. The non-linear problem is re-formulated in terms of the appropriate Green's function in the next section.

\section{GREEN'S FUNCTION METHODOLOGY}

The infinite dilution PB problem of an isolated platelet immersed with counterions in an electrolyte solution has been solved numerically using a finite difference scheme [17]. However, this method is not well adapted to the case of a charged platelet confined to a WS cell. Here a semi-analytic method is proposed, whereby the PB equation is transformed into a non-linear integral equation, which can be solved iteratively. This approach requires the knowledge of a Green's function $G\left(\mathbf{r}, \mathbf{r}^{\prime}\right)$ such that its "convolution" product with the charge density $\rho_{c}(\mathbf{r})$, defined by Eqs. (5) and (㺼), namely

$$
-\frac{4 \pi}{\epsilon}\left(G * \rho_{c}\right)(\mathbf{r}) \equiv-\frac{4 \pi}{\epsilon} \int_{v} G\left(\mathbf{r}, \mathbf{r}^{\prime}\right) \rho_{c}\left(\mathbf{r}^{\prime}\right) d \mathbf{r}^{\prime}
$$

satisfies Poisson's equation (3) and the Neumann boundary condition (2); $\left(G * \rho_{c}\right)$ coincides then with the required potential $\varphi(\mathbf{r})$. Note that the integral in Eq. (10) is not, strictly speaking, a convolution since $G$ is not a function of the relative position $\mathbf{r}-\mathbf{r}^{\prime}$ only.

By definition, the Green's function is a solution of the following linear Poisson's equation

$$
\nabla_{\mathbf{r}}^{2} G\left(\mathbf{r}, \mathbf{r}^{\prime}\right)=\delta\left(\mathbf{r}-\mathbf{r}^{\prime}\right) .
$$

Integrating both sides of Eq. (11), and using Gauss' theorem,

$$
\begin{aligned}
\int_{v} \nabla_{\mathbf{r}}^{2} G\left(\mathbf{r}, \mathbf{r}^{\prime}\right) d \mathbf{r} & =\int_{\Sigma} \mathbf{n}(\mathbf{r}) \cdot \nabla_{\mathbf{r}} G\left(\mathbf{r}, \mathbf{r}^{\prime}\right) d S \\
& =\int_{v} \delta\left(\mathbf{r}-\mathbf{r}^{\prime}\right) d \mathbf{r}=1,
\end{aligned}
$$

it becomes clear that the Green's function satisfying (11) cannot obey the same boundary condition (2) as the potential, but rather

$$
[\mathbf{n}(\mathbf{r}) \cdot \nabla]_{\mathbf{r} \in \Sigma} G\left(\mathbf{r}, \mathbf{r}^{\prime}\right)=\frac{1}{\mathcal{S}(\Sigma)}
$$

where $\mathcal{S}(\Sigma)$ is the total area of the WS cell. The boundary condition (13) precludes the possibility of expanding $G$ in a Bessel-Dini series [19], similar to that used for the solution of the LPB problem in the same cylindrical geometry [15,16. The difficulty may be overcome by adopting one of three possible routes.

a) Eq. (11) is solved subject to the boundary condition

$$
[\mathbf{n}(\mathbf{r}) \cdot \nabla]_{\mathbf{r} \in \Sigma}\left(G * \rho_{c}\right)(\mathbf{r})=0 .
$$

An explicit example is given in Appendix A.

b) A modified Green's function $G^{\mathcal{B}}$ is sought, which satisfies the following Poisson's equation

$$
\nabla_{\mathbf{r}}^{2} G^{\mathcal{B}}\left(\mathbf{r}, \mathbf{r}^{\prime}\right)=\delta\left(\mathbf{r}-\mathbf{r}^{\prime}\right)+\mathcal{B}(\mathbf{r})
$$

where $\mathcal{B}(\mathbf{r})$ is an arbitrary neutralising background charge distribution, such that

$$
\int_{v} \mathcal{B} d \mathbf{r}=-1
$$


$G^{\mathcal{B}}\left(\mathbf{r}, \mathbf{r}^{\prime}\right)$ can now be required to satisfy the same boundary condition (2) as the potential. It is easily verified that $-4 \pi\left(G^{\mathcal{B}} * \rho_{c}\right) / \epsilon$ indeed satisfies Eq. (3), together with the appropriate Neumann boundary condition (2), since

$$
\begin{aligned}
\nabla^{2}\left[-\frac{4 \pi}{\epsilon}\left(G^{\mathcal{B}} * \rho_{c}\right)\right] & =-\frac{4 \pi}{\epsilon} \int_{v} \nabla_{\mathbf{r}}^{2} G^{\mathcal{B}}\left(\mathbf{r}, \mathbf{r}^{\prime}\right) \rho_{c}\left(\mathbf{r}^{\prime}\right) d \mathbf{r}^{\prime} \\
& =-\frac{4 \pi}{\epsilon} \rho_{c}(\mathbf{r})-\frac{4 \pi}{\epsilon} \mathcal{B}(\mathbf{r}) \int_{v} \rho_{c}\left(\mathbf{r}^{\prime}\right) d \mathbf{r}^{\prime} \\
& =-\frac{4 \pi}{\epsilon} \rho_{c}(\mathbf{r})
\end{aligned}
$$

where overall charge neutrality of the WS cell has been used; similarly

$$
[\mathbf{n}(\mathbf{r}) \cdot \nabla]_{\mathbf{r} \in \Sigma}\left(G^{\mathcal{B}} * \rho_{c}\right)(\mathbf{r})=\int_{v}\left\{[\mathbf{n}(\mathbf{r}) \cdot \nabla]_{\mathbf{r} \in \Sigma} G^{\mathcal{B}}\left(\mathbf{r}, \mathbf{r}^{\prime}\right)\right\} \rho_{c}\left(\mathbf{r}^{\prime}\right) d \mathbf{r}^{\prime}=0 .
$$

in view of the boundary condition imposed on $G^{\mathcal{B}}$.

c) The bare Laplace operator in Eq. (11) is replaced by a dressed or screened operator, and the solution $G^{\kappa}$ of

$$
\left(\nabla_{\mathbf{r}}^{2}-\kappa^{2}\right) G\left(\mathbf{r}, \mathbf{r}^{\prime}\right)=\delta\left(\mathbf{r}-\mathbf{r}^{\prime}\right)
$$

can then be made to satisfy the Neumann boundary condition (2) for any non-zero value of the inverse length $\kappa$. The solution of this linear problem may be obtained analytically along the same lines as those leading to the potential within LPB theory [15,16], as sketched in Appendix A. Subtracting $\kappa^{2} \varphi(\mathbf{r})$ from both sides of the non-linear PB equation (9), it is then straightforward to check that the solution to that equation, subject to the proper boundary condition (2), satisfies the non-linear integral equation

$$
\varphi(\mathbf{r})=-\frac{4 \pi}{\epsilon} \int_{v} G^{\kappa}\left(\mathbf{r}, \mathbf{r}^{\prime}\right)\left(q_{P}\left(\mathbf{r}^{\prime}\right)+e\left[\rho_{0}^{+} \mathrm{e}^{-\beta e \varphi\left(\mathbf{r}^{\prime}\right)}-\rho_{0}^{-} \mathrm{e}^{\beta e \varphi\left(\mathbf{r}^{\prime}\right)}\right]+\kappa^{2} \frac{\epsilon}{4 \pi} \varphi\left(\mathbf{r}^{\prime}\right)\right) d \mathbf{r}^{\prime}
$$

Since $G^{\kappa}\left(\mathbf{r}, \mathbf{r}^{\prime}\right)$ is known analytically, Eq. (20) may be solved numerically by an iterative procedure, starting from an initial guess for $\varphi(\mathbf{r})$.

Details for the analytic solutions of Eqs. (11), (15) and (19), subject to the appropriate boundary conditions, are given in Appendix A. In practice the iterative procedure was implemented numerically using route c). Using the results from Appendix A, the required Green's function may be written in the form of the following Bessel-Dini series

$$
G^{\kappa}\left(\mathbf{r}, \mathbf{r}^{\prime}\right)=\sum_{n \geq 1} \mathcal{C}_{n}^{ \pm}\left(\phi, \mathbf{r}^{\prime}\right) J_{0}\left(y_{n} \frac{r}{R}\right) \cosh \left[\frac{h \mp z}{\Lambda_{n}}\right]
$$

where $\mathbf{r}=(r, \phi, z)$ (cylindrical coordinates), the signs + and - correspond to the situations $z>z^{\prime}$ and $z<z^{\prime}$ respectively, $y_{n}$ is the $\mathrm{n}^{\text {th }}$ root of $J_{1}(y)=0, J_{0}$ and $J_{1}$ are the zeroth and first order cylindrical Bessel functions, and $\Lambda_{n}^{-2}=\left(y_{n}^{2}+\kappa^{2} R^{2}\right) / R^{2}$. The coefficients $\mathcal{C}_{n}^{ \pm}\left(\phi, \mathbf{r}^{\prime}\right)$ are given by

$$
\mathcal{C}_{n}^{ \pm}\left(\phi, \mathbf{r}^{\prime}\right)=-2 \Lambda_{n} \frac{\delta\left(\phi-\phi^{\prime}\right)}{R^{2} \sinh \left[2 h / \Lambda_{n}\right]} \frac{J_{0}\left(y_{n} r^{\prime} / R\right)}{J_{0}^{2}\left(y_{n}\right)} \cosh \left[\frac{h \pm z^{\prime}}{\Lambda_{n}}\right] .
$$

For an infinitely thin platelet of radius $r_{0}$, the charge density $q_{P}(\mathbf{r})$, which provide the source term in Poisson's equation, may be simply expressed as

$$
q_{P}(\mathbf{r})=\sigma \Theta\left(r_{0}-r\right) \delta(z)
$$

where $\Theta$ is the Heaviside function. The corresponding contribution to $\varphi(\mathbf{r})$ in Eq. (20) may then be evaluated analytically

$$
\int_{v} d \mathbf{r}^{\prime} G^{\kappa}\left(\mathbf{r}, \mathbf{r}^{\prime}\right) q_{P}\left(\mathbf{r}^{\prime}\right)=-\sigma \frac{r_{0}}{R} \sum_{n \geq 1}^{\infty} \Lambda_{n} \frac{J_{1}\left(y_{n} r_{0} / R\right)}{y_{n} \sinh \left[h / \Lambda_{n}\right]} \frac{J_{0}\left(y_{n} r / R\right)}{J_{0}^{2}\left(y_{n}\right)} \cosh \left[\frac{h \mp z}{\Lambda_{n}}\right]
$$


The non-linear integral equation (20) was then solved for $\varphi(\mathbf{r})$ using an iterative Picard method with underrelaxation [20]. At a particular iteration step $i$, the result from the previous iteration, $\varphi^{i-1}(\mathbf{r})$, and the analytic result (24) are used as input to compute the r.h.s of Eq. (20). The resulting potential $\varphi(\mathbf{r})$ is then used to produce the input for the next iteration step by mixing it with $\varphi^{i-1}(\mathbf{r})$ according to

$$
\varphi^{i}(\mathbf{r})=\alpha \varphi^{i-1}(\mathbf{r})+(1-\alpha) \varphi(\mathbf{r})
$$

where $0<\alpha<1$; typically $\alpha \approx 0.9$. The iterative cycle is repeated until the relative difference $\left|\varphi^{i}-\varphi^{i-1}\right| /\left|\varphi^{i}\right|$ at the centre of the cylindrical WS cell (which coincides with the centre of the disc) becomes smaller than a pre-set value, chosen to be $10^{-5}$ in practice.

The arbitrary inverse length $\kappa$ is chosen in the range $\left[\kappa_{D} / 10,10 \kappa_{D}\right]$, where $\kappa_{D} \equiv\left(8 \pi n_{S}^{\prime} e^{2} /\left(\epsilon k_{B}\right)^{T}\right)^{1 / 2}$ is the inverse Debye length in the reservoir, in the case of calculations carried out in the semi-grand-canonical ensemble. The prefactors $\rho_{0}^{+}$and $\rho_{0}^{-}$in Eq. (5) are fixed during the calculation at values obeying the constraint (8). Note that the electroneutrality condition (1) is not obeyed until the iterations have converged to the actual solution $\varphi(\mathbf{r})$, thus providing an additional global convergence test. If the calculations are carried out in the canonical ensemble, $n_{S}=n_{-}$ is fixed, and $\kappa_{D} \equiv\left[8 \pi n_{S} e^{2} /\left(\epsilon k_{B} T\right)\right]^{1 / 2}$. The concentration of counterions is fixed by the electroneutrality condition (11) and the prefactors $\rho_{0}^{+}$and $\rho_{0}^{-}$are determined by Eq. (7) at each step of the iteration, so that electroneutrality holds throughout the convergence process.

The converged solution $\varphi(\mathbf{r})$ must be independent of the particular choice of the auxiliary variable $\kappa$. Cylindrical symmetry implies $\varphi(\mathbf{r})=\varphi(r, z)$, which is calculated on a 2-dimensional grid $n_{r} \times n_{z}$ spanning half the cylinder. In practice $n_{r}=240$ grid points were used to cover the interval $[0, R]$, which provides sufficient resolution for the representation of the Bessel functions, and $n_{z}=100$ points for the interval $[0, h]$. 60 terms were retained in the Bessel-Dini series (21). Starting from the initial guess $\varphi(\mathbf{r}) \equiv 0$, convergence is generally achieved in about 50 to 200 iterations. The number of iterations needed to achieve a relative accuracy of $10^{-5}$ can be drastically reduced if the electrostatic potential obtained from the hybrid theory, introduced in the next section, is used as input.

\section{A HYBRID POISSON-BOLTZMANN/DEBYE-HÜCKEL THEORY}

The hybrid Poisson-Boltzmann/Debye-Hückel (PB/DH) theory was developed for the problem of a clay platelet in a cylindrical WS cell as a first attempt to go beyond LPB [15]. Within this symmetry the potential $\varphi(\mathbf{r})=\varphi(r, z)$ can be expanded in a Bessel-Dini series [19]

$$
\beta e \varphi(z, r)=\sum_{n=1}^{\infty} A_{n}(z) J_{0}\left(\frac{y_{n} r}{R}\right)
$$

which factorizes the dependence of the potential on the distance $z$ along the axis of the cylinder, and the radial distance $r \equiv\left(x^{2}+y^{2}\right)^{1 / 2}$. The hybrid $\mathrm{PB} / \mathrm{DH}$ approach is an attempt to treat edge effects in a perturbative way, whilst keeping the non-linear PB description in the limit of infinite platelets.

Substituting (26) into Eq. (5), the leading term $A_{1}(z)$ in the expansion is exponentiated, but the exponential is linearised with respect to the remainder of the series $(n \geq 2)$

$$
\rho^{ \pm}=\rho_{0}^{ \pm} \mathrm{e}^{\mp A_{1}(z)}\left(1 \mp \sum_{n=2}^{\infty} A_{n}(z) J_{0}\left(y_{n} \frac{r}{R}\right)\right) .
$$

The first term in this expansion, $n=1$, corresponds to the solution of the PB equation for an infinite charged plane in a WS slab of height $2 h$, with an effective surface charge $\sigma^{\prime}=\sigma\left(r_{0} / R\right)^{2}$. The terms of order $n \geq 2$ may be considered as providing an estimate of the correction to the infinite charged plane limit $\left(r_{0} \rightarrow R\right)$ due to the finite size of the platelets [15].

The concentrations of co and counterions are determined by

$$
n^{ \pm}=\rho_{0}^{ \pm} \frac{1}{2 h} \int_{-h}^{+h} e^{\mp A_{1}(z)} d z
$$

It is therefore impossible to impose $\rho_{0}^{+}=n^{+}$and $\rho_{0}^{-}=n^{-}$simultaneously. As a consequence, in the presence of added salt, calculations are preferably performed in the semi-grand canonical ensemble, at fixed reservoir salt concentration. This can be achieved with the choice 


$$
\rho_{0}^{+}=\rho_{0}^{-}=n_{S}^{\prime} .
$$

For $z \neq 0$ the semi-linearised Poisson-Boltzmann equation becomes

$$
\begin{aligned}
\nabla^{2} \varphi(\mathbf{r})= & -\frac{4 \pi e}{\epsilon} \times \\
& \left\{\rho_{0}^{+} \mathrm{e}^{-A_{1}(z)}-\rho_{0}^{-} \mathrm{e}^{A_{1}(z)}-\left(\rho_{0}^{+} \mathrm{e}^{-A_{1}(z)}+\rho_{0}^{-} \mathrm{e}^{A_{1}(z)}\right) \sum_{n \geq 2}^{\infty} A_{n}(z) J_{0}\left(y_{n} \frac{r}{R}\right)\right\} .
\end{aligned}
$$

The boundary condition associated with the presence of the uniformly charged platelet at $z=0, r<r_{0}$ is

$$
\left(\frac{\partial \varphi(z, r)}{\partial z}\right)_{z=0^{+}}=-\frac{2 \pi \sigma}{\epsilon} \Theta\left(r_{0}-r\right)
$$

The electric field normal to the top and bottom of the cylinder vanishes, i.e.

$$
\left(\frac{\partial \varphi(z, r)}{\partial z}\right)_{z= \pm h}=0
$$

Using (29) in (30) and then projecting on the basis of the zeroth order Bessel functions $J_{0}$ leads to the following set of differential equations

$$
\begin{gathered}
\frac{d^{2} A_{1}(z)}{d z^{2}}=8 \pi l_{B} n_{S}^{\prime} \sinh A_{1}(z) \\
\frac{d^{2} A_{n}(z)}{d z^{2}}-\left(\frac{y_{n}}{R}\right)^{2} A_{n}(z)=8 \pi l_{B} n_{S}^{\prime} A_{n}(z) \sinh A_{1}(z) \quad, n \geq 2
\end{gathered}
$$

with the boundary conditions at $z=0$

$$
\begin{aligned}
& \left(\frac{d A_{1}(z)}{d z}\right)_{z=0^{+}}=-\frac{1}{b}\left(\frac{r_{0}}{R}\right)^{2} \\
& \left(\frac{d A_{n}(z)}{d z}\right)_{z=0^{+}}=-\frac{2}{b}\left(\frac{r_{0}}{R}\right) \frac{1}{y_{n} J_{n}^{2}\left(y_{n}\right)} J_{1}\left(\frac{y_{n} r_{0}}{R}\right) \quad, n \geq 2
\end{aligned}
$$

and at $z= \pm h$

$$
\left(\frac{d A_{n}(z)}{d z}\right)_{z= \pm h}=0 \quad, n \geq 1
$$

where $l_{B}=\beta e^{2} / \epsilon$ is the Bjerrum length and $b=e / 2 \pi \sigma l_{B}$ the Gouy length.

This set of differential equations can be solved analytically in the salt-free case, for a WS cylindrical cell of infinite height 15] ( $h \rightarrow \infty, R$ finite). A numerical solution must be sought otherwise. The first equation in (33) is identical to the non-linear PB equation for the ion distribution in a 1-dimensional cell model where each platelet is assumed to be an infinite uniformly charged plane confined with its monovalent co and counterions to a slab. In order to solve this equation we follow the prescription given in Appendix B of [5]. A first order differential equation can be obtained after integrating once, using the boundary condition at $z=h$

$$
\frac{d A_{1}(z)}{d z}=-\sqrt{16 \pi l_{B} n_{S}^{\prime}\left(\cosh A_{1}(z)-\cosh A_{1}(h)\right)} .
$$

The equation above is solved numerically using a 4th order Runge-Kutta algorithm. Different guesses for $A_{1}(h)$ lead to different solutions $A_{1}(z)$. An underestimated initial guess for $A_{1}(h)$ is increased until a solution $A_{1}(z)$ that verifies the boundary condition at $z=0$ is found.

The second equation in (33) can also be solved by first reducing it to a first order differential equation and then using an appropriate numerical algorithm for its integration. Defining a function

$$
L(z) \equiv 8 \pi l_{B} n_{S}^{\prime} \sinh A_{1}(z)-\left(\frac{y_{n}}{R}\right)^{2},
$$


one obtains

$$
\frac{d^{2} A_{n}(z)}{d z^{2}}-L(z) A_{n}(z)=0
$$

which is a stiff differential equation if $L(z)$ is large. This can be reduced to a first order differential equation by applying a Ricatti transform

$$
\eta(z)=\frac{1}{A_{n}(z)} \frac{d A_{n}(z)}{d z}
$$

which leads to

$$
\frac{d \eta(z)}{d z}=L(z)-\eta(z)^{2}
$$

The differential equation above is again solved using a 4th order Runge-Kutta algorithm, and forced to satisfy the boundary condition at $z=h$ re-written as

$$
\eta(h)=0
$$

Integration of (39) leads to

$$
A_{n}(z)=C \exp \left[\int_{0}^{z} \eta\left(z^{\prime}\right) d z^{\prime}\right]
$$

The integral above can be evaluated numerically while the integration constant $C$ is determined by the boundary condition at $z=0$

$$
C=\frac{1}{\eta(0)}\left(\frac{d A_{n}(z)}{d z}\right)_{z=0} .
$$

The Bessel-Dini coefficients $A_{n}(z)$ in the expansion (26) of the electrostatic potential can be computed numerically using the procedure just described. This involves solving as many independent differential equations as terms kept in the expansion, $i e$ one for each coefficient. The computational cost is considerably reduced compared to the solution of the non-linear PB equation using the iterative scheme prescribed in the previous section.

\section{RESULTS}

\section{A. Potential and density profiles}

Figs. 17a-c compare the dimensionless electrostatic potential $\Phi(z, r) \equiv \beta e \varphi(z, r)$ as obtained within LPB [15, 16], $\mathrm{PB} / \mathrm{DH}$ and PB. The PB/DH potential was computed by first solving (33) for the coefficients $A_{n}(z)$ and then using these in the Bessel-Dini expansion (26) for the electrostatic potential. Only the projections $(z=0, r)$ and $(z, r=0)$ are shown. The results are for an aqueous solution of clay discs of radius $r_{0}=150 \AA$ at temperature $T=300 \mathrm{~K}$. The aspect ratio of the cylindrical WS cell is $h / r_{0}=1.25$ and the concentration of clay $n=5 \times 10^{-3} \mathrm{M}(1 \mathrm{M}=1 \mathrm{~mol}$ $\mathrm{dm}^{-3}$ ). Although in the present model the clay platelets are assumed to be infinitely thin, a packing fraction can be defined by assining a finite thickness $d$ to the platelets; $d$ can be chosen to be $1 \mathrm{~nm}$, as appropriate for Laponite [14].

The $\mathrm{PB}$ and $\mathrm{PB} / \mathrm{DH}$ results in Fig. 目 are semi-grand canonical calculations for $n_{S}^{\prime}=5 \times 10^{-3} \mathrm{M}$ and $Z=5,100$ and 1000. The Donnan ratios $n_{S} / n_{S}^{\prime}$ in Table 1 compare well, indicating that the concentration of added salt in the WS cell is approximately the same in $\mathrm{PB}$ and $\mathrm{PB} / \mathrm{DH}$, for a fixed $Z$. Unlike other thermodynamic quantities (see next section), this macroscopic property is well estimated within the $\mathrm{PB} / \mathrm{DH}$ theory.

The LPB results of Fig. 1 have been produced for the same salt concentrations $n_{S}$ in the cell as those obtained within the semi-grand canonical PB theory (see Table [i). Once $n_{S}$ is known, the Donnan ratio reads within LPB [21]

$$
n_{S} / n_{S}^{\prime}=\left(1+Z n / n_{S}\right)^{-1 / 2} .
$$

The corresponding data are collected in Table $\mathrm{f}$. The Donnan ratio in LPB is smaller that in PB and the difference increases with $Z$. For a given salt concentration in the reservoir, LPB theory underestimates the concentration of added salt in the WS cell. 
Turn next to the comparison of the electrostatic potentials in Fig. 1. Although at very low surface charge $(Z=5)$ all theories produce an identical electrostatic potential, as soon as $Z$ is as large as 100, it becomes obvious that the linearisation of the Boltzmann factors is no longer an acceptable approximation. However, at such high surface charge densities, the $\mathrm{PB} / \mathrm{DH}$ still provides a very good approximation for $\varphi(z, r)$. At physically relevant charge densities, e.g. $Z=1000$, the LPB approximation gives rise to an electrostatic potential substantially different from that obtained within $\mathrm{PB}$ or $\mathrm{PB} / \mathrm{DH}$. The $\mathrm{PB} / \mathrm{DH}$ theory still yields a good approximation for the potential except near the surface of the platelet, for $r \gtrsim r_{0}$. This illustrates the breakdown of linearisation to account for finite size corrections.

Up to a rescaling factor, the shapes of PB and LPB potentials are similar (Fig. 11). For a given charge density on the platelets (fixed $Z$ ), one can then define an effective charge $Z^{*}$ from a maximum likelyhood criterion between $\varphi^{P B}(Z)$ and $\varphi^{L P B}\left(Z^{*}\right)$ : for $Z=1000$ and the parameters of Fig. 目, the corresponding effective charge is $Z^{*}=375$ (see Fig. 2). As expected, $Z^{*}<Z$, which means that the counterions recondense within a thin layer around the particle until the electric potential near the surface is lowered to a value of a few $k_{B} T$. Fig. 2 shows that the resulting agreement is only qualitative and that the renormalized LPB potential can neither account for the edge effects nor for the behaviour along the $z$ axis. However, in the vicinity of the WS surface and far from the platelet where the variations of the potential are weak, PB theory can be linearised and the potential retains an LPB form, provided the bare charge is replaced by an effective charge $Z^{*}$. In the simpler spherical geometry, given the non-linear PB electric potential, Alexander et al. 11] proposed to determine the effective parameters for the linearised theory by matching to the far-field limit of the PB solution: the resulting LPB potential, matching the PB potential up to its third derivative, is then an effective potential for the colloid-colloid interactions. The phase diagram associated with the latter effective interactions was later shown to be in good agreement with experiments for charged spherical colloids [22]. In the case of non-spherical colloids in anisotropic cells, the condensation of micro-ions may well be non uniform so that the effective particle may have a different charge distribution than the original one. Preliminary results confirm this scenario for clay platelets: the effective charge $Z^{*}$ depends on the point chosen on the WS surface to match the LPB and PB potentials, except of course when $Z$ is low enough. The concept of charge renormalization thus seems to be inadequate for clay particles due to the non uniformity of micro-ions condensation.

\section{B. Osmotic properties and quadrupole moment}

Once the potential $\varphi(\mathbf{r})$ and the concentration profiles $\rho^{+}(\mathbf{r})$ and $\rho^{-}(\mathbf{r})$ have been determined within $\mathrm{PB}, \mathrm{PB} / \mathrm{DH}$ or LPB theories, a number of osmotic properties may be calculated, as discussed in detail in Refs. [15, 16]. The Helmholtz free energy is obtained by substituting the profiles into the functional (6), with the result

$$
\beta F=\beta\left(U_{P}-U_{C}\right)+\left(N^{+}+N^{-}\right)\left[\ln \left(n_{S}^{\prime} \lambda^{3}\right)-1\right] .
$$

where $\lambda$ is an irrelevant length scale, and $U_{P}$ and $U_{C}$ are the following two contributions to the internal energy $U=U_{P}+U_{C}$

$$
U_{P}=\frac{1}{2} \int_{v} q_{P}(\mathbf{r}) \varphi(\mathbf{r}) d \mathbf{r}, \quad U_{C}=\frac{e}{2} \int_{v}\left[\rho^{+}(\mathbf{r})-\rho^{-}(\mathbf{r})\right] \varphi(\mathbf{r}) d \mathbf{r} .
$$

Expression (45) is valid within the semi-grand canonical ensemble (which was used throughout), with the choice of $\rho_{0}^{+}=\rho_{0}^{-}=n_{S}^{\prime}$. The resulting grand potential is

$$
\beta \Omega=\beta F-2 N^{-} \ln \left(n_{S}^{\prime} \lambda^{3}\right) .
$$

For a given clay concentration, and hence for a given WS volume $v, \Omega$ and all osmotic properties depend on the aspect ratio $h / R$ (or equivalently $h / r_{0}$ ). The equilibrium topology of the columnar stacking of platelets, as represented by the WS cell model, is that which minimizes $\Omega$ as a function of $h / r_{0}$, for given values of $v, n_{S}^{\prime}, T$ and $\epsilon$. For convenience, the results shown in the figures are shifted by $\Omega_{0} \equiv Z \ln \left(n_{S}^{\prime} \lambda^{3}\right)$, ie

$$
\beta\left(\Omega-\Omega_{0}\right)=\beta\left(U_{P}-U_{C}\right)+N^{-}+N^{+} .
$$

The osmotic pressure $\Pi$ can be derived from the pressure tensor [15, 16]

$$
\Pi=\frac{1}{\beta} \sum_{k=+,-}{\overline{\rho^{k}}}^{\Sigma}+\frac{\epsilon}{8 \pi}{\overline{(\nabla \varphi)^{2}}}^{\Sigma},
$$

where $\overline{(. .)}^{\Sigma}$ denotes an average over the total surface $\Sigma$ of the WS cell. The osmotic pressure in the reservoir is $\Pi_{0} \equiv 2 k_{B} T n_{S}^{\prime}$. For a cylindrical WS cell a disjoining pressure can be defined by 


$$
\Pi_{d}=\frac{1}{\beta} \sum_{k=+,-}{\overline{\rho^{k}}}^{S^{\prime}}+\frac{\epsilon}{8 \pi} \overline{|\nabla \varphi|^{2}} S^{\prime}
$$

where $S^{\prime} \subset \Sigma$ is the surface of the bottom and top of the WS cell. The swelling arises from the osmotic pressure exerted by the micro-ions and a normal spacing between the platelets (equal to the height of the cylindrical cell) is obtained when an axial pressure $\Pi_{d}$ is applied.

The a priori lowest order non-vanishing multipole moment of the charge distribution in the WS cell is the $z z$ component of the traceless quadrupole tensor

$$
Q_{z z}^{t o t}=\frac{1}{2} \int_{v} d \mathbf{r}\left[\rho_{c}(\mathbf{r})\right]\left(2 z^{2}-x^{2}-y^{2}\right) .
$$

Upon integration by parts, this may be re-expressed as a surface integral

$$
Q_{z z}^{t o t}=\frac{\epsilon}{4 \pi} \frac{1}{2} \oint_{\Sigma} \varphi(\mathbf{r}) \nabla\left(2 z^{2}-x^{2}-y^{2}\right) \cdot \mathbf{d} \mathbf{S},
$$

where Poisson's equation has been used. Numerical accuracy of the solution $\varphi(\mathbf{r})$ to the PB equation can be checked by comparing the quadrupole as obtained from the two equations (51) and (52). In the plots, $Q_{z z}^{t o t}$ is normalised by the quadrupole moment of the disc

$$
\begin{aligned}
Q_{z z}^{d i s c} & =\frac{1}{2} \int_{v} q_{P}(\mathbf{r})\left(2 z^{2}-x^{2}-y^{2}\right) d \mathbf{r} \\
& =\frac{Z e r_{0}^{2}}{4} .
\end{aligned}
$$

Figs. 3a-c show results for the osmotic and disjoining pressures, grand potential and quadrupole moment as functions of the aspect ratio $h / r_{0}$, for $Z=100$. Results from $\mathrm{PB}$ and $\mathrm{PB} / \mathrm{DH}$ are compared. Within the latter theory, the concentration profiles may be calculated from Eq. (27), once the coefficients $A_{n}$ have been obtained; alternatively, the profiles may be calculated by re-exponentiating the potential determined from Eq. (26), according to Eq. (5). Fig. 3a shows that the osmotic pressure $\Pi$ goes through a minimum at a well-defined aspect ratio. Within PB theory, the disjoining pressure $\Pi_{d}$ coincides with the osmotic pressure at that minimum. The re-exponentiated version of $\mathrm{PB} / \mathrm{DH}$ theory leads to results in reasonable agreement with the $\mathrm{PB}$ data, whereas straight $\mathrm{PB} / \mathrm{DH}$ theory yields an unphysical osmotic pressure which is lower than the reservoir pressure $\Pi_{0}$; note that although the corresponding disjoining pressure agrees well with the $\mathrm{PB}$ data, it intersects the osmotic pressure curve away from the minimum in the latter, and at a much higher aspect ratio. Fig. $3 \mathrm{~b}$ shows that the PB results for the grand potential $\Omega$ go through a flat minimum at the same aspect ratio as the osmotic pressure. The minimum is shifted to considerably higher aspect ratios within $\mathrm{PB} / \mathrm{DH}$ theory and its re-exponentiated form, and does not coincide with the corresponding minima in the osmotic pressure curves. The PB quadrupole moments calculated from Eqs. (51) and (52) coincide and go through zero at the value of $h / r_{0}$ corresponding to the osmotic pressure and grand potential minima of the Figs 3a,b. The PB/DH results are fairly close to their PB counterparts but go through zero at an aspect ratio which is significantly lower than the location of the minimum of the corresponding grand potential. The re-exponentiated $\mathrm{PB} / \mathrm{DH}$ results, on the other hand, as calculated from Eqs. (51) and (52) are inconsistent.

Calculations at the very low platelet charge $Z=5$ lead to undistinguishable results between the PB and reexponentiated $\mathrm{PB} / \mathrm{DH}$ theories, as one might expect from the nearly identical potential profiles in Fig. 1. However, at the physically relevant charge $Z=1000$, the discrepancies between the two theories become very large, as illustrated in Fig. 1 .

The scenario emerging from PB theory is reminiscent of the predictions of LPB theory. In the framework of the latter the Helmholtz free energy goes through a minimum at the same value of the aspect ratio $h / r_{0}$ for which the disjoining and osmotic pressures are equal. The quadrupole moment $Q$ vanishes at this same $h / r_{0}$. The same behaviour follows from the present PB results in the semi-grand canonical ensemble, showing that $Q_{z z}^{\text {tot }}=0$ and $\Pi=\Pi_{d}$ at the aspect ratio that minimises the grand potential (see Figs. 3 and 1 ). This illustrates the robustness of the equivalence between

- a thermodynamic minimization

- an electrostatic criterion $\left(Q_{z z}^{t o t}=0\right)$

- a mechanical equilibrium condition $\left(\Pi=\Pi_{d}\right)$ 
- the minimization of an osmotic constraint: $\Pi$ is minimum at the aspect ratio where $\Pi=\Pi_{d}$ (see Fig. Ba and ta. Also holds within LPB).

The equilibrium separation between two platelets is determined by the minimisation of the grand potential (or Helmholtz free energy).

Being an approximate theory for the electrostatic potential, the $\mathrm{PB} / \mathrm{DH}$ is inconsistent at $Z \gtrsim 100$. However, in spite of all the deficiencies which the $\mathrm{PB} / \mathrm{DH}$ theory exhibits when trying to compute macroscopic quantities from the electrostatic potential, this approximation does manage to give a good first approximation for $\varphi(\mathbf{r})$ which compares well with that obtained by solving the PB equation.

\section{FORCE ACTING BETWEEN TWO PARALLEL PLATELETS}

The Green's function methodology of section [II may be extended to calculate the potential and density profiles around two coaxial platelets placed symmetrically inside a WS cell, within PB theory. If the two uniformly charged parallel discs are placed at $z= \pm z_{0}$ from the centre of a WS cell as shown in Fig. 同, the PB equation is still given by (9), with the source term $q_{P}(\mathbf{r})$ now including a contribution from both platelets

$$
q_{P}(\mathbf{r})=\sigma \Theta\left(r_{0}-r\right)\left[\delta\left(z+z_{0}\right)+\delta\left(z-z_{0}\right)\right] .
$$

The boundary conditions (2) still applies and the integral equation (20) for the local potential $\varphi(\mathbf{r})$ remains unchanged, with the contribution (24) from the source term now following from (21) and (54), with the result

$$
\int_{v} d \mathbf{r}^{\prime} G^{\kappa}\left(\mathbf{r}, \mathbf{r}^{\prime}\right) q_{P}\left(\mathbf{r}^{\prime}\right)=-2 \sigma \frac{r_{0}}{R} \sum_{n \geq 1}^{\infty} \Lambda_{n} \frac{J_{1}\left(y_{n} r_{0} / R\right)}{y_{n} \sinh \left[h / \Lambda_{n}\right]} \frac{J_{0}\left(y_{n} r / R\right)}{J_{0}^{2}\left(y_{n}\right)} \Upsilon(z)
$$

where the function $\Upsilon(z)$ is given by

$$
\Upsilon(z)= \begin{cases}\cosh \left[z_{0} / \Lambda_{n}\right] \cosh \left[(h-z) / \Lambda_{n}\right] & , z_{0}<z \\ \cosh \left[z / \Lambda_{n}\right] \cosh \left[\left(h-z_{0}\right) / \Lambda_{n}\right] & ,-z_{0}<z<z_{0} \\ \cosh \left[z_{0} / \Lambda_{n}\right] \cosh \left[(h+z) / \Lambda_{n}\right] & , z<-z_{0} .\end{cases}
$$

An example of a PB potential profile under conditions appropriate for Laponite is shown in Fig. 6 .

Once $\varphi(\mathbf{r})$ and the resulting concentration profiles $\rho^{\alpha}(\mathbf{r})$ are known, the local stress tensor may be evaluated at each point of the WS cell

$$
\begin{aligned}
& \overleftrightarrow{\boldsymbol{\Pi}}(\mathbf{r})=p(\mathbf{r}) \overleftrightarrow{\mathbf{I}}-\frac{\varepsilon}{4 \pi} \nabla \varphi \otimes \nabla \varphi, \\
& p(\mathbf{r})=k_{B} T \sum_{\alpha=+,-} \rho^{\alpha}(\mathbf{r})+\frac{\varepsilon}{8 \pi}|\nabla \varphi(\mathbf{r})|^{2}
\end{aligned}
$$

where $\stackrel{\leftrightarrow}{\mathbf{I}}$ denotes the unit tensor. The force acting on platelet $i \in(1,2)$ follows by integrating the stress tensor (57) over both sides, $\Sigma_{\mathcal{P}, i}^{+}$and $\Sigma_{\mathcal{P}, i}^{-}$, of the platelet

$$
\mathbf{F}_{i}=-\int_{\Sigma_{\mathcal{P}, i}^{+} ; \Sigma_{\mathcal{P}, i}^{-}} \overleftrightarrow{\Pi} \cdot \mathbf{d} \mathbf{S}_{i}
$$

In the case under consideration, of two identical coaxial discs, the force $\mathbf{F}_{1}=-\mathbf{F}_{2}$ is along the unit vector $\hat{\mathbf{z}}$ of the cylinder axis. The electric field $\mathbf{E}=-\boldsymbol{\nabla} \varphi$ in the vicinity of platelet $i$ may be decomposed into continuous and discontinuous parts

$$
\mathbf{E}=\mathbf{E}_{i}^{(d)}+\mathbf{E}_{i}^{(c)}= \pm \frac{2 \pi}{\varepsilon} \sigma \mathbf{n}_{i}+\mathbf{E}_{i}^{(c)} .
$$

The force $\mathbf{F}_{i}$ may then also be expressed as

$$
\mathbf{F}_{i}=\sigma \int_{\Sigma_{\mathcal{P}, i}} \mathbf{E}_{i}^{(c)} d^{2} S \quad(i=1,2)
$$


where the integration runs over the surface of platelet $i$. The numerical evaluation of the force from (61) poses technical difficulties associated with the removal of the discontinuity suffered by the electric field across the platelet which requires some care (cf. Appendix B).

Returning to the definition (59), the surface integral may be transformed using the mechanical equilibrium condition

$$
\nabla \cdot \overleftrightarrow{\Pi}(\mathbf{r})=\mathbf{0}
$$

into the following integral over the surface $S$, enclosing the upper half of the WS cell (cf. Fig. 5)

$$
\mathbf{F}_{1}=-\mathbf{F}_{2}=-\int_{S} \overleftrightarrow{\Pi} \cdot \mathbf{d} \mathbf{S}
$$

Because $\mathbf{n} \cdot \boldsymbol{\nabla} \varphi=0$ on the outer surface $\Sigma$ of the WS cell, and $\partial \varphi(\mathbf{r}) / \partial z=0$ by symmetry on the cross-section of the cylinder at $z=0$, the term $\boldsymbol{\nabla} \varphi \otimes \nabla \varphi$ gives a vanishing contribution to the force $\mathbf{F}_{1}$, which reduces to

$$
\mathbf{F}_{1}=-\int_{S} p(\mathbf{r}) \mathbf{d} \mathbf{S}
$$

This may be further simplified by noting that $\hat{\mathbf{z}} \cdot \mathbf{n}=0$ on the lateral surface of the cylinder (parallel to the axis), so that finally

$$
F_{1}^{z}=\int_{z=0} p(\mathbf{r}) d S-\int_{z=h} p(\mathbf{r}) d S .
$$

The numerical consistency between results based on Eq. (65) and on Eq. (61) (cf. Appendix B) has been carefully checked. Explicit calculations were carried out in the limit of vanishing clay concentration $(n \rightarrow 0)$. This was achieved by choosing a WS cylindrical cell large enough for the local electric field to vanish before the outer surface of the cell is reached. For platelets with the physical characteristics of Laponite $\left(r_{0}=150 \AA\right.$ and $\left.Z=1000\right)$, and salt concentrations $n_{S} \gtrsim 10^{-3} \mathrm{M}$, this condition is met with a cell volume $v=4 \times 10^{8} \AA^{3}$ (corresponding to a clay concentration $n=8.3 \times 10^{-6} \mathrm{M}$ ) and an aspect ratio $h / r_{0}=1.5$. A test that the WS cell was chosen sufficiently large is provided by checking that the calculated concentration $n_{S}$ of salt in the cell coincides with the preset reservoir concentration $n_{S}^{\prime}$, as expect for vanishing clay concentration. The forces calculated within PB theory are plotted versus the distance between platelets in Fig. 7, and compared to the analytical prediction of LPB theory [16]

$$
F^{z}(d)=\left(\pi r_{0}\right)^{2} \frac{4 \pi \sigma^{2}}{\epsilon} \int_{0}^{\infty} J_{1}^{2}(x) \frac{1}{x} \exp \left[-\frac{d}{r_{0}} \sqrt{x^{2}+\kappa_{D}^{2} r_{0}^{2}}\right] .
$$

For $Z=5$ the PB and LPB results coincide, but in the physically relevant case $Z=1000$ shown in Fig. 7, LPB theory overestimates the force by an order of magnitude. This finding is consistent with the overestimation of the absolute value of the potential around a platelet by LPB theory, as illustrated in Fig. 17c. Note that unlike its LPB counterpart, the $\mathrm{PB}$ force does not decay exponentially with the distance between platelets.

\section{CONCLUSION}

Swollen stacks of monodisperse clay platelets are conveniently modelled within a Wigner-Seitz cell representation. Relevant electrostatic and thermodynamic properties are derived from the inhomogeneous counterion and coion concentration profiles related to the local electrostatic potential by Poisson's equation. Adopting the primitive model point of view for the solvent and neglecting spatial correlations between macroions leads to the closed PB equation for the potential $\varphi(\mathbf{r})$ which is solved subject to Neumann boundary conditions on the confining surface of the WS cell. Whilst the linearised LPB version of the theory yields to analytic treatment [15, 16], the present paper concerned with the numerical solution of the full non-linear PB problem and of a hybrid $\mathrm{PB} / \mathrm{DH}$ version of the theory. Rather than solving the two-dimensional non-linear partial differential PB equation on a grid, it was found that a more adequate and stable method is to reduce the problem to a non-linear integral equation for $\varphi(\mathbf{r})$, involving an electrostatic Green's function satisfying appropriate boundary conditions. Three equivalent routes are proposed, each involving a Green's function which may be calculated explicitly in terms of a Bessel-Dini series, similar to that used for the solution of the LPB problem.

While the present results generally confirm the qualitative trends predicted by the LPB analysis [15] 16], there are considerable quantitative differences at physically relevant surface charge densities of the Laponite platelets. The main findings may be summarized as follows 
a) LPB theory overestimates the magnitude of the local potential by typically a factor of two for a platelet of $10^{3} \mathrm{e}$.

b) Rescaling of the platelet charge to a lower effective value to force good agreement of the PB and LPB potentials at the centre of the platelet fails because the shapes of the potential profiles differ significantly, particularly near edges.

c) The scenario of the variation of various properties with the WS aspect ratio, for a fixed value of the cell volume, derived from non-linear PB theory confirms the LPB predictions: the grand potential (or the Helmholtz free energy) goes through a minimum at a well-defined aspect ratio $h / r_{0}$; at this same aspect ratio, the osmotic pressure is also at its minimum, where it coincides with the monotonically decreasing disjoining pressure, while the quadrupole moment of the charge distribution within the WS cell vanishes. Thus the same equilibrium aspect ratio is selected by thermodynamic, mechanical, osmotic and electrostatic criteria.

d) The hybrid $\mathrm{PB} / \mathrm{DH}$ theory, which is linearised with respect to edge effects yields rather accurate potential profiles, but rather poor thermodynamic properties; the agreement with full PB theory is improved upon reexponentiation of the Bessel-Dini series for the local potential.

e) The same Green's function methodology allows an accurate calculation of the force between the two coaxial platelets. The force calculated within PB theory is an order of magnitude smaller than the LPB prediction, and decreases with the distance between the platelets in a non-exponential fashion, up to distances of the order of the radius $r_{0}$ of the platelets.

In future work it is planned to include microion correlations and discrete solvent (hydration) effects, by generalising the PB functional (6), along the lines proposed by Biben et al. [3]. Generalisation of the present methodology to flexible charged membranes would also be helpful in describing charged soap films or smectite clay particles of lateral dimensions larger than those of Laponite.

\section{Acknowledgments}

E.T. thanks J.O. Fossum, G. Manificat, T. Nicolai and F. van Wijland for interesting discussions. R.J.F.L. de C. has carried out work at the ENS de Lyon as part of a project financed by the European Commission through the Training and Mobility of Researchers (TMR) programme. He is now at UCL, funded by NERC. The present collaboration was facilitated by a grant from the British-French Alliance Programme.

\begin{tabular}{|c|c|c|c|}
\hline & $Z=5$ & $Z=100$ & $Z=1000$ \\
\hline \multicolumn{4}{|c|}{$n_{S} / n_{S}^{\prime}$} \\
\hline LPB & 0.975 & 0.648 & 0.233 \\
\hline $\mathrm{PB} / \mathrm{DH}$ & 0.976 & 0.720 & 0.564 \\
\hline PB & 0.976 & 0.725 & 0.574 \\
\hline \multicolumn{4}{|c|}{$n_{S} / 10^{-3} \mathrm{M}$} \\
\hline $\mathrm{LPB}$ and $\mathrm{PB}$ & 4.878 & 3.623 & 2.870 \\
\hline PB/DH & 4.881 & 3.602 & 2.820 \\
\hline
\end{tabular}

TABLE I. Values of the Donnan ratios $n_{s} / n_{s}^{\prime}$ obtained within LPB, PB/DH or PB theory, for an aqueous solution of clay concentration $n=5 \times 10^{-5} \mathrm{M}$. The temperature is $300 \mathrm{~K}$ and the radius of the discs $r_{0}=150 \AA$. The reservoir salt concentration in $\mathrm{PB}$ and $\mathrm{PB} / \mathrm{DH}$ is $n_{s}^{\prime}=5 \times 10^{-3} \mathrm{M}$. The concentrations of added salt in the WS cell are also indicated. 


\section{APPENDIX A:}

For the three routes outlined in section [IV, $G\left(G^{\mathcal{B}}\right.$ in case $\mathbf{b}$ or $G^{\kappa}$ in case $\left.\mathbf{c}\right)$ is expanded in a Bessel-Dini series

$$
G\left(\mathbf{r}, \mathbf{r}^{\prime}\right)=\sum_{n \geq 1} G_{n}\left(\phi, z, \mathbf{r}^{\prime}\right) J_{0}\left(y_{n} \frac{r}{R}\right)
$$

Note that the coefficients of any expansion

$$
f(r)=\sum_{n \geq 1} f_{n} J_{0}\left(y_{n} \frac{r}{R}\right)
$$

can be obtained from the inversion relation

$$
f_{n}=\frac{2}{R^{2} J_{0}^{2}\left(y_{n}\right)} \int_{0}^{R} r f(r) J_{0}\left(y_{n} \frac{r}{R}\right) d r
$$

so that the Dirac distribution inside the WS cell can be cast in the form

$$
\begin{aligned}
\delta\left(\mathbf{r}-\mathbf{r}^{\prime}\right) & \equiv \frac{1}{r} \delta\left(r-r^{\prime}\right) \delta\left(\phi-\phi^{\prime}\right) \delta\left(z-z^{\prime}\right) \\
& =\sum_{n \geq 1} \frac{2}{R^{2} J_{0}^{2}\left(y_{n}\right)} J_{0}\left(y_{n} \frac{r}{R}\right) J_{0}\left(y_{n} \frac{r^{\prime}}{R}\right) \delta\left(\phi-\phi^{\prime}\right) \delta\left(z-z^{\prime}\right)
\end{aligned}
$$

Consider specifically route c. Subsitution of Eqs. (A4) and (A1) into (19) yields

$$
\frac{d^{2} G_{n}}{d z^{2}}-\frac{\Lambda_{n}^{2}}{R^{2}} G_{n}=\frac{2 J_{0}\left(y_{n} r^{\prime} / R\right)}{R^{2} J_{0}^{2}\left(y_{n}\right)} \delta\left(\phi-\phi^{\prime}\right) \delta\left(z-z^{\prime}\right)
$$

where $\Lambda_{n}^{-2}=y_{n}^{2} / R^{2}+\kappa^{2}$. With the boundary condition

$$
\left.\frac{d G_{n}}{d z}\right|_{z= \pm h}=0
$$

the solution reads

$$
G_{n}\left(\phi, z, \mathbf{r}^{\prime}\right)=\mathcal{C}_{n}^{ \pm} \cosh \left(\frac{h \mp z}{\Lambda_{n}}\right)
$$

where the superscripts + and - refer to the situations $z>z^{\prime}$ and $z<z^{\prime}$ respectively. The coefficients $\mathcal{C}_{n}^{ \pm}$are conveniently obtained by writing $G_{n}$ in the form

$$
G_{n}\left(\phi, z, \mathbf{r}^{\prime}\right)=g_{n}^{+}(z, \phi) \Theta\left(z-z^{\prime}\right)+g_{n}^{-}(z, \phi) \Theta\left(z^{\prime}-z\right)
$$

Invoking the identity

$$
f(z) \frac{d}{d z} \delta\left(z-z^{\prime}\right)=-f^{\prime}(z) \delta\left(z-z^{\prime}\right)+f\left(z^{\prime}\right) \frac{d}{d z} \delta\left(z-z^{\prime}\right)
$$

the continuity conditions obeyed by $G_{n}$ at $z=z^{\prime}$ follow as

$$
\begin{aligned}
& g_{n}^{+}\left(z^{\prime}\right)=g_{n}^{-}\left(z^{\prime}\right) \\
& \frac{d}{d z} g_{n}^{+}\left(z^{\prime}\right)-\frac{d}{d z} g_{n}^{-}\left(z^{\prime}\right)=\frac{2}{R^{2} J_{0}^{2}\left(y_{n}\right)} J_{0}\left(y_{n} \frac{r^{\prime}}{R}\right) \delta\left(\phi-\phi^{\prime}\right),
\end{aligned}
$$

leading back to Eq. (22)

An example of Green's function associated with method a can be obtained by imposing

$$
\left.\frac{d G_{n}}{d z}\right|_{z= \pm h}=0, \quad \text { for } n \geq 2
$$


while the non-Neumann character of $G$ reflects itself in the boundary condition obeyed by $G_{1}$, that follows from the resolution of A5 with $\kappa=0$. One finds

$$
G_{1}\left(z, \phi, \mathbf{r}^{\prime}\right)=\frac{1}{R^{2}} \delta\left(\phi-\phi^{\prime}\right)\left|z-z^{\prime}\right|
$$

whereas the remaining terms $G_{n}, n \geq 2$ are the same as in (21) and (22), with $\kappa=0$.

Finally, method $\mathbf{b}$ can be illustrated along similar lines with the following choice of the background

$$
\mathcal{B}=-\frac{1}{h R^{2}} \delta\left(\phi-\phi^{\prime}\right)
$$

The above density is such that the expansion (21), with relation (22) and $\kappa=0$, is unchanged for $n \geq 2$ while the first term is now slightly modified with respect to Eq. (A12), namely

$$
G_{1}\left(z, \phi, \mathbf{r}^{\prime}\right)=\left[\frac{1}{R^{2}}\left|z-z^{\prime}\right|-\frac{1}{2 h R^{2}}\left(z^{2}+z^{\prime 2}\right)\right] \delta\left(\phi-\phi^{\prime}\right) .
$$

\section{APPENDIX B:}

From Eq. (61), the force acting on platelet 1 sitting at $z=+z_{0}$ can be written

$$
F_{1}^{z}=-2 \pi \sigma \int_{0}^{r_{0}} d r r\left[\left(\frac{\partial \varphi(z, r)}{\partial z}\right)_{z=z_{0}} \pm \frac{2 \pi}{\epsilon} \sigma\right],
$$

where - is for $z=z_{0}^{+}$and + is for $z=z_{0}^{-}$. This equation allows the immediate computation of the force from the electrostatic potential. However, it should not be used directly since the numerical differentiation of the potential for $z=z_{0}$ and $0<r<r_{0}$ (where $\partial \varphi(\mathbf{r}) / \partial z$ is discontinuous) is inaccurate.

Instead, apply Gauss' theorem to (B1) to obtain

$$
\begin{aligned}
F^{z} & =\sigma \frac{Q_{1}}{\epsilon}-2 \pi \sigma \int_{r_{0}}^{R} d r r\left(\frac{\partial \varphi(z, r)}{\partial z}\right)_{z=z_{0}}-\frac{Z e \sigma}{2 \epsilon} \\
& =-\sigma \frac{Q_{2}}{\epsilon}-2 \pi \sigma \int_{r_{0}}^{R} d r r\left(\frac{\partial \varphi(z, r)}{\partial z}\right)_{z=z_{0}}+\frac{Z e \sigma}{2 \epsilon},
\end{aligned}
$$

where $Q_{1}$ is the total charge (excluding the platelet) in the WS cell volume limited by $z_{0}<z<h$ and $Q_{2}$ is the total charge in the volume of the WS cell limited by $0<z<z_{0}$

$$
\begin{aligned}
& Q_{1}=2 \pi \int_{z_{0}^{+}}^{h} d z \int_{0}^{R} d r r \rho_{c}(\mathbf{r}) \\
& Q_{2}=2 \pi \int_{0}^{z_{0}^{-}} d z \int_{0}^{R} d r r \rho_{c}(\mathbf{r}) .
\end{aligned}
$$

Due to the imposed electroneutrality $Q_{1}$ and $Q_{2}$ verify

$$
Q_{1}+Q_{2}=e Z
$$

Eq. (B2) requires the computation of the derivative of the electrostatic potential at $z=z_{0}$ and $r_{0}<r<R$, where $\partial \varphi(\mathbf{r}) / \partial z$ is continuous. The differentiation can be accurately performed by fitting $\varphi(z, r)$ to a polynomial in $z$, at fixed $r$. 
[1] G. Gouy, J. Physique 9, 457 (1910).

[2] D. I. Chapman, Phil. Mag. 25, 475 (1913).

[3] A. T. Augousti amd G. Rickayzen, J. Chem. Soc. Faraday Trans. 2 80, 141 (1984); V. Kralj-Iglic and A. Iglic, J. Phys. II 6, 477 (1996); T. Biben, J.-P. Hansen and Y. Rosenfeld, Phys. Rev. E 57 R3727 (1998).

[4] D. Andelman, in Membranes, their Structure and Conformations, edited by R. Lipowsky and E. Sackmann (Elsevier, Amsterdam, 1996).

[5] M. Dubois, T. Zemb, L. Belloni L., A. Delville, P. Levitz and R. Setton, J. Chem. Phys. 96, 2278 (1992).

[6] B. V. Derjaguin and L. Landau, Acta Physiochim. (USSR) 14, 633 (1941).

[7] E. J. Verwey and J. Th. G. Overbeek, Theory of the Stability of Lyotropic Colloids (Elsevier, Amsterdam, 1948).

[8] O. Stern, Z. Elektrochem. 30, 508 (1924).

[9] R. M. Fuoss, A. Katchalsky and S. Lifson, Proc. Nat. Acad. Sci. U.S. 37, 579 (1951).

[10] G. S. Manning, J. Chem. Phys. 51, 924 (1969).

[11] See, e.g., S. Alexander, P. M. Chaikin, P. Grant, J. M. Morales, P. Pincus and D. Hone, J. Chem. Phys. 80, 5776 (1984).

[12] T. Gisler, S. F. Schulz, M. Bokovec, H. Sticker, P. Schurtenberger, B. d'Aguanno and R. Klein, J. Chem. Phys. 101, 9924 (1994).

[13] J. C. Crocker and D. G. Grier, Phys. Rev. Letters 77, 1897 (1996).

[14] Laporte Inorganics, Laponite Technical Bulletin L104/90/A.

[15] E. Trizac and J.-P. Hansen, J. Phys. Cond. Matter 9, 2683 (1997).

[16] E. Trizac and J.-P. Hansen, Phys. Rev. E 56, 3137 (1997).

[17] R. B. Secor and C. J. Radke, J. Colloid Interface Sci. 103, 237 (1985); F. R. C. Chang and G. Sposito, ibid. 163, 19 (1994).

[18] R. J. F. Leote de Carvalho, E. Trizac and J.-P. Hansen, Europhys. Lett. 43, 369 (1998).

[19] G. N. Watson, A Treatise on the Theory of Bessel Functions, 2nd ed. (Cambridge University Press, 1995).

[20] A. A. Broyles, J. Chem. Phys 33, 456 (1960).

[21] J.-P. Hansen and E. Trizac, Physica A 235, 257 (1997).

[22] T. Palberg, W. Mönch, F. Bitzer, R. Piazza and T. Bellini, Phys. Rev. Letters 74, 4555 (1995). 
(a)

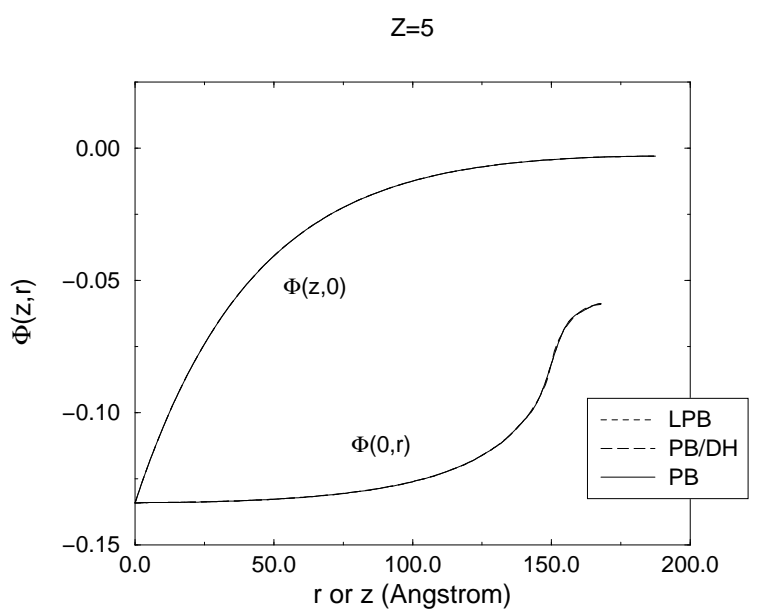

(b)

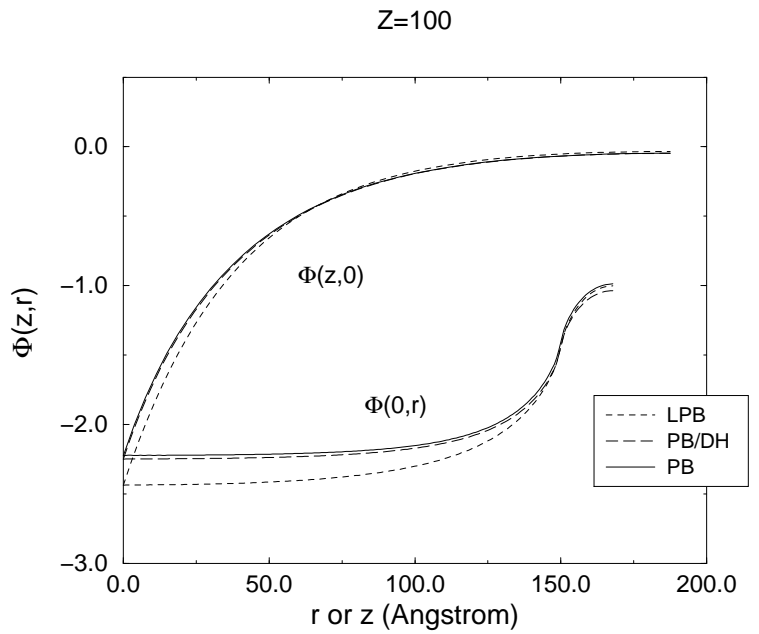

(c)

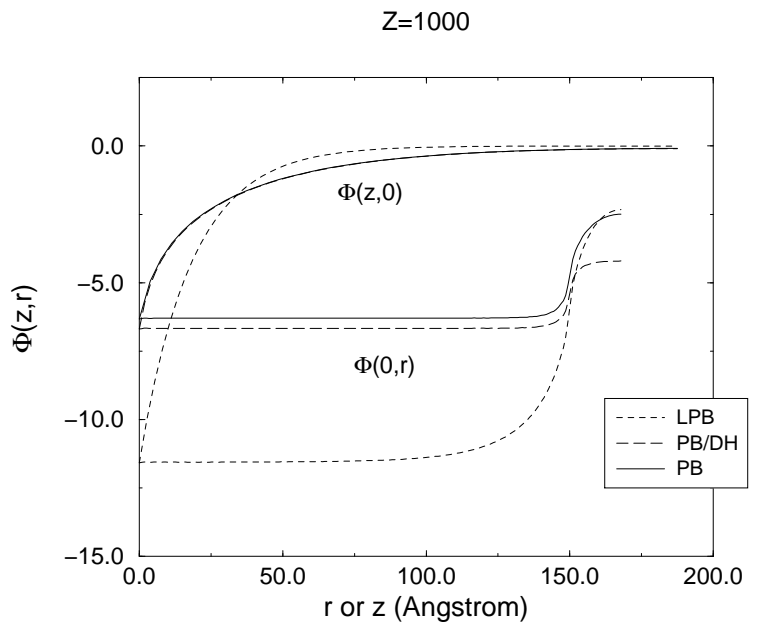

FIG. 1. Dimensionless electrostatic potential $\Phi(z, r) \equiv \beta e \varphi(z, r)$ at $z=0$ versus $r$, and at $r=0$ versus $z$. The profiles were obtained in LPB (dotted line), in PB/DH (dashed line) and in the full non-linear PB theories (solid line). (See text for details.) The surface charge on the clay discs is: (a) $Z=5$, (b) $Z=100$ and (c) $Z=1000$. The concentrations of added salt and the Donnan ratios $n_{S} / n_{S}^{\prime}$ for these results are indicated in Table I. 


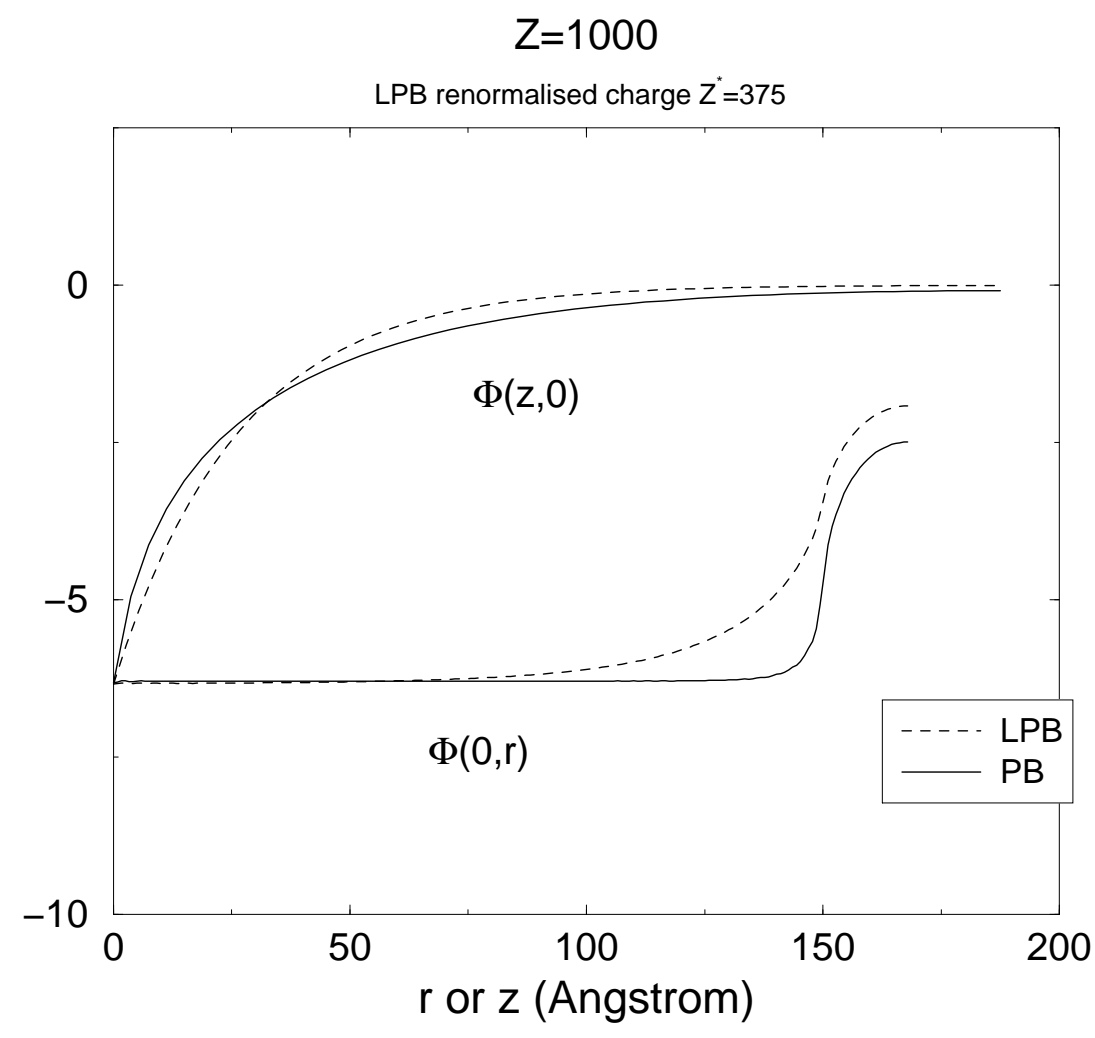

FIG. 2. Comparison between the non-linear PB potential for $Z=1000$ (as in Figure 1 ) and the linearised LPB potential, now with an effective renormalised charge $Z^{*}=375$. 
(a)

(b)
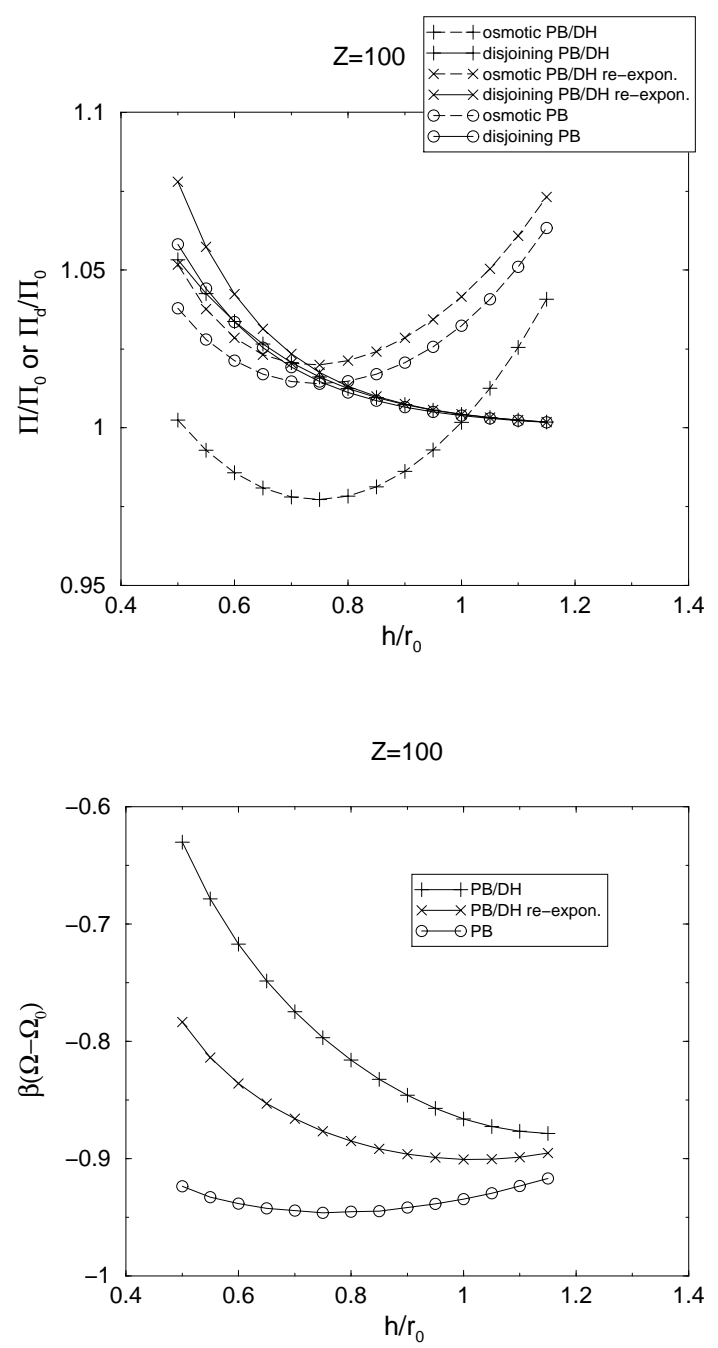

(c)

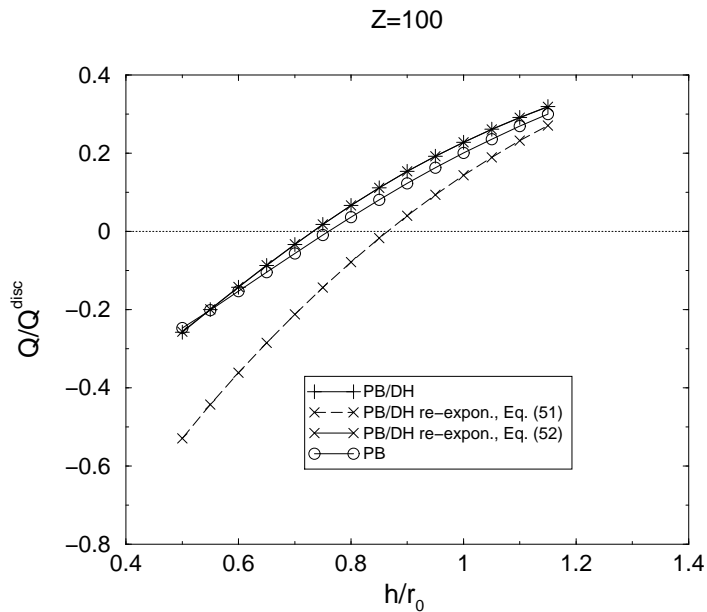

FIG. 3. Results obtained within the $\mathrm{PB}$ and $\mathrm{PB} / \mathrm{DH}$ theories for an aqueous solution of clay discs of charge $Z=100$ and radius $r_{0}=150 \AA$. The clay concentration is $n=5 \times 10^{-5} \mathrm{M}$ and $T=300 K$. The $\circ$ are $\mathrm{PB}$ results, + are results from $\mathrm{PB} / \mathrm{DH}$ theory and $\times$ are from $\mathrm{PB} / \mathrm{DH}$ with re-exponentiation. In (a) the osmotic pressure, $\Pi$, is represented by dashed lines and the disjoining pressure, $\Pi_{d}$, by solid lines. (b) shows the grand potential while (c) shows the quadrupole moment. The quadrupole obtained within $\mathrm{PB} / \mathrm{DH}$ with re-exponentiation is not consistent: different results are obtained from Eqs. (51) (solid line) and (52) (dashed line). 
(a)

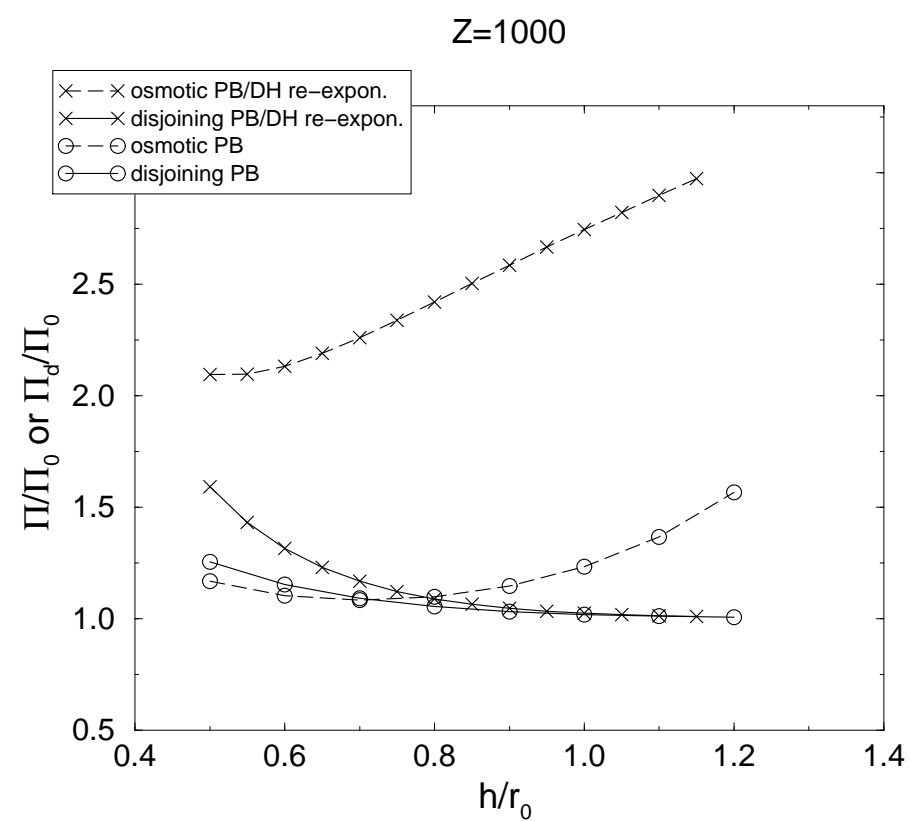

(b)

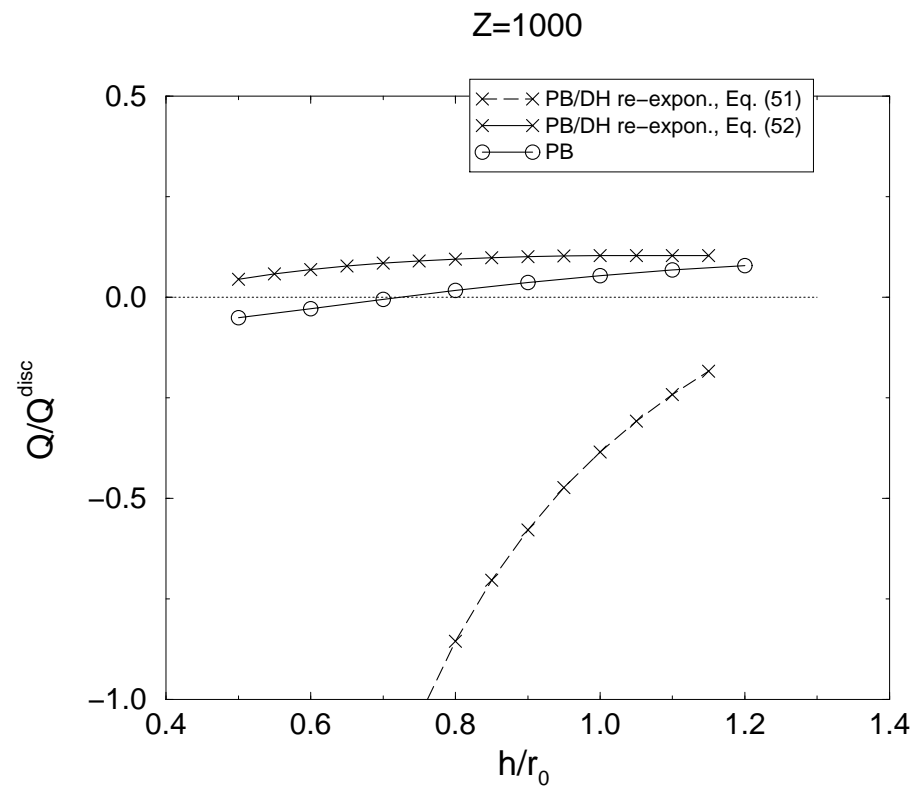

FIG. 4. Results obtained within the $\mathrm{PB}$ and $\mathrm{PB} / \mathrm{DH}$ theory with re-exponentiation for an aqueous solution of clay discs of charge $Z=1000$ and radius $r_{0}=150 \AA$. The clay concentration is $n=5 \times 10^{-5} \mathrm{M}$ and $T=300 K$. The $\circ$ are $\mathrm{PB}$ results and $\times$ are from $\mathrm{PB} / \mathrm{DH}$ with re-exponentiation. In (a) the solid lines are disjoining pressures and the dashed lines are osmotic pressures. (b) shows the quadrupole moment. Different results for the quadrupole are obtained within PB/DH with re-exponentiation, from Eqs. (51) (solid line) and (52) (dashed line). 


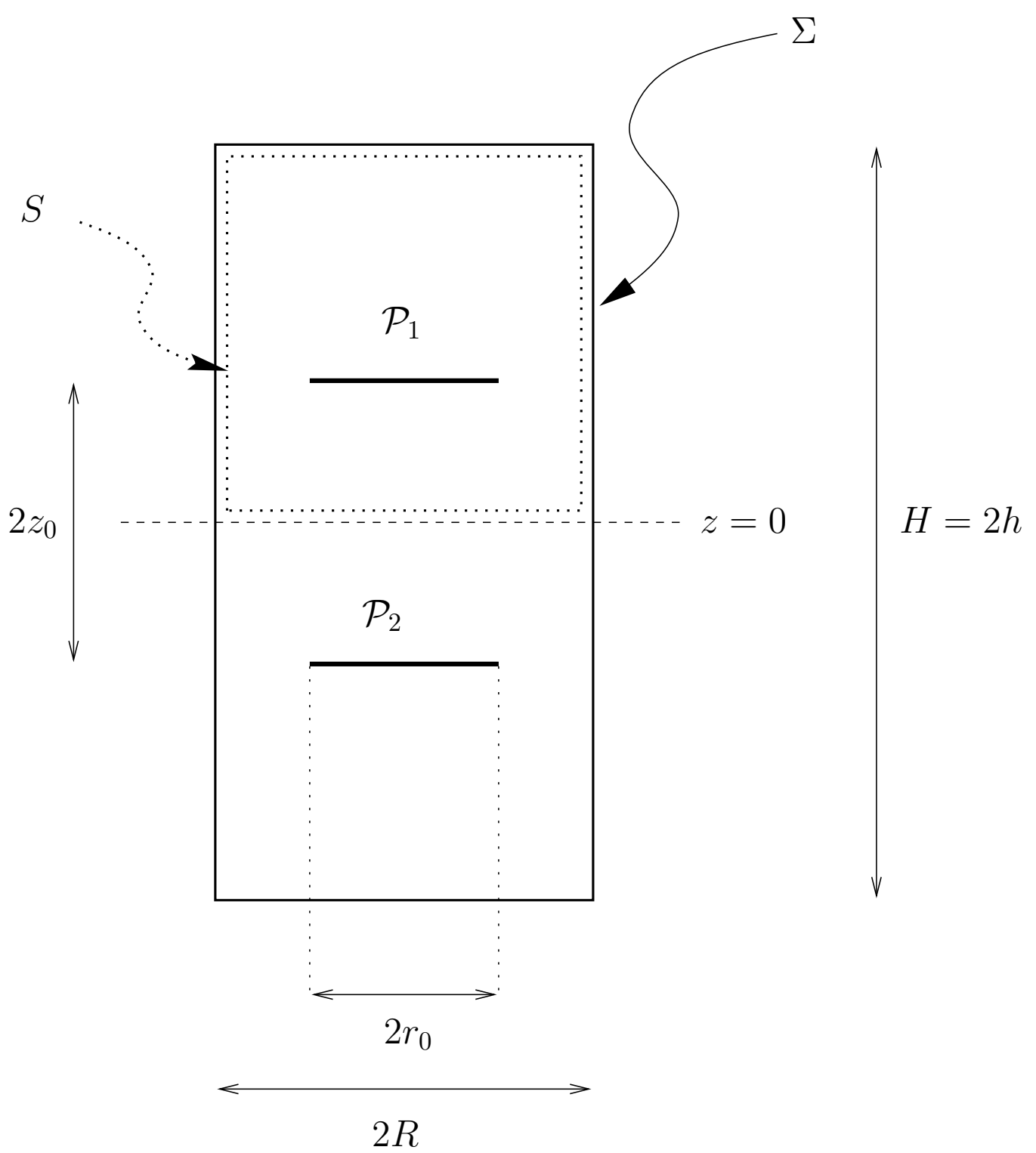

FIG. 5. Side view of the Wigner-Seitz cylinder associated with the 2 platelets problem 


\section{$Z=1000$}

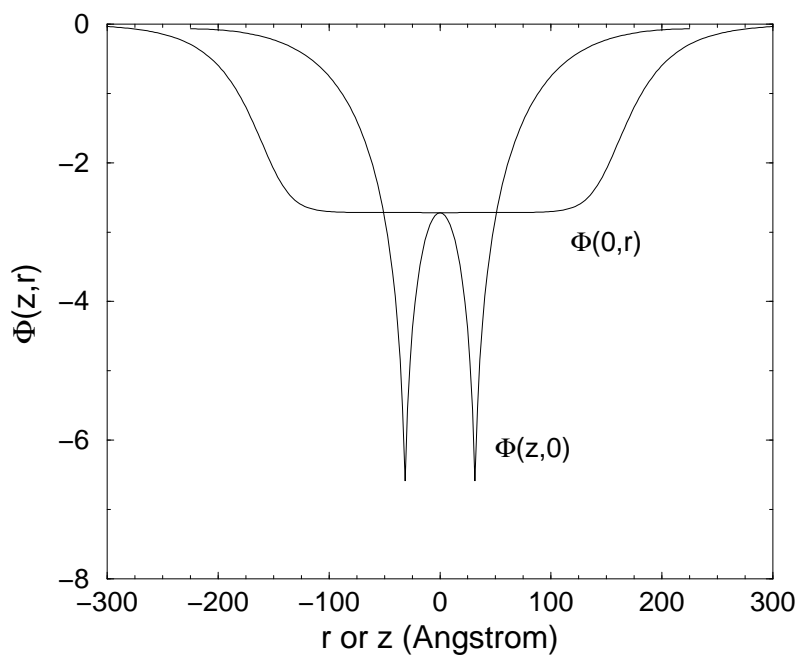

FIG. 6. Dimensionless electrostatic potential $\Phi(z, r) \equiv \beta e \varphi(z, r)$ at $z=0$ versus $r$ and at $r=0$ versus $z$. The profiles were obtained by solving the PB equation for an aqueous solution of two Laponite discs $\left(Z=1000, r_{0}=150 \AA\right)$ at a temperature $T=300 \mathrm{~K}$. The distance between the two discs is $d=0.24 r_{0}$, the height of the cylindrical WS cell is $h=1.5 r_{0}$ and the aspect ratio $h / R=0.423$. The concentration of monovalent added salt in the WS cell is $n_{S}=4.64 \times 10^{-3} \mathrm{M}$.

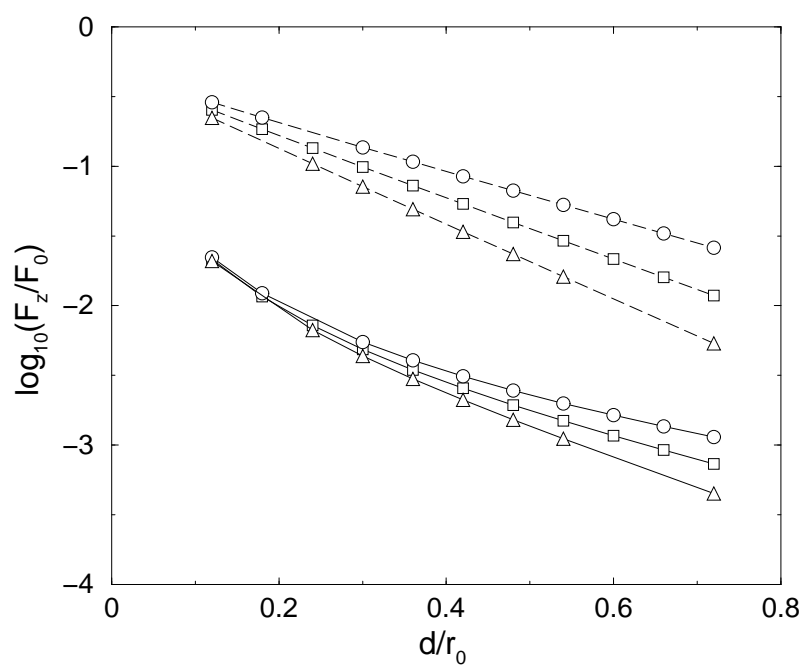

FIG. 7. The force between two parallel platelets of Laponite as a function of the distance separating their coaxial centres $\left(F_{0}=4 \pi^{2} r_{0}^{2} \sigma^{2} / \epsilon\right)$. The solid lines are results obtained from non-linear PB and the dashed lines from LPB. The $\bigcirc$ are for $n_{S}=0.00078 \mathrm{M}, \square$ for $n_{S}=0.0046 \mathrm{M}$, and the $\triangle$ for $n_{S}=0.0095 \mathrm{M}$. 